University of Wollongong

Research Online

Faculty of Engineering - Papers (Archive)

Faculty of Engineering and Information

Sciences

August 2006

\title{
Power generation with nanowire resonant tunneling thermoelectrics
}

Mark F. O'Dwyer

University of Wollongong, markod@uow.edu.au

H. Linke

University of Oregon, USA

E. Hoffmann

University of Oregon, USA

T. E. Humphrey

University of Geneva, Switzerland

R. A. Lewis

University of Wollongong, roger@uow.edu.au

See next page for additional authors

Follow this and additional works at: https://ro.uow.edu.au/engpapers

Part of the Engineering Commons

https://ro.uow.edu.au/engpapers/365

\section{Recommended Citation}

O'Dwyer, Mark F.; Linke, H.; Hoffmann, E.; Humphrey, T. E.; Lewis, R. A.; and Zhang, C.: Power generation with nanowire resonant tunneling thermoelectrics 2006.

https://ro.uow.edu.au/engpapers/365

Research Online is the open access institutional repository for the University of Wollongong. For further information contact the UOW Library: research-pubs@uow.edu.au 
Authors

Mark F. O'Dwyer, H. Linke, E. Hoffmann, T. E. Humphrey, R. A. Lewis, and C. Zhang 


\title{
Power generation with nanowire resonant tunneling thermoelectrics
}

\author{
M. F. O’Dwyer ${ }^{1}$, H. Linke ${ }^{2}$, E. Hoffmann ${ }^{2}$, T. E. Humphrey ${ }^{3}$, R. A. Lewis ${ }^{1}$ and C. Zhang ${ }^{1}$ \\ ${ }^{1}$ School of Engineering Physics and Institute for Superconducting and Electronic Materials \\ University of Wollongong, Wollongong NSW 2522, Australia \\ ${ }^{2}$ Materials Science Institute and Physics Department, University of Oregon, Eugene, OR 97403-1274, U.S.A. \\ ${ }^{3}$ Département de Physique Théorique, University of Geneva, CH-1211, Geneva, Switzerland
}

\begin{abstract}
Nanowires have been predicted to yield significantly improved thermoelectric figures of merit. Here we describe the electron energy spectrum of a resonant tunneling heterostructure nanowire in detail and show how it may be used to realize energy filtering for thermoelectric electronic efficiencies approaching the Carnot limit. A proof-ofprinciple experiment is described that seeks to demonstrate such idealized electronic properties.
\end{abstract}

\section{Introduction}

Whereas low efficiencies limit the practical application of current thermoelectric devices to niche applications, a breakthrough in thermoelectric technology allowing higher efficiencies would have substantial economic impact. Recent theory predicts that the use of nanowires in thermoelectric devices could have significant advantages. Hicks and Dresselhaus predicted considerably higher thermoelectric figures of merit for $\mathrm{Bi}_{2} \mathrm{Te}_{3}$ based nanowires compared to superlattice or bulk devices primarily due to changes in the electronic density of states [1]. Of particular interest here is the prediction that low-dimensional conductors in which mobile electrons are restricted to a very narrow range can achieve electronic energy-conversion efficiencies approaching the Carnot limit $[2,3]$.

Here, we discuss in detail the requirements for a nanowire thermoelectric device to realize efficiency approaching the Carnot limit and develop specific structural parameters for an InAs/InP nanowire heterostructure to achieve these. The device described is a wire with a single embedded quantum dot, through which electrons tunnel via a resonant transmission energy between hot and cold electron reservoirs. Such a device might be used in a proof-of-principle experiment demonstrating that electron energy filtering in $1 \mathrm{D}$ conductors can significantly increase the efficiency of thermoelectric energy conversion.

\section{Nanowires overview}

Lithographic methods have traditionally been used to make quantum devices, however, such methods have drawbacks [4]. Semiconductor whiskers were first grown and characterized in the 1960s with diameters in the order of a hundred nanometers or more [5]. Although different growth mechanisms exist, all have been described using the vaporliquid-solid mechanism [5]. Here a metal cluster, such as Au, acts as a catalyst under which the whisker grows [6].

In the 1990s whiskers with diameters in the order of tens of nanometers were grown, providing quantum confinement for new devices [7]. Since then rapid progress has occurred both in nanowire growth and the development of nanowire devices. Nanowires have been grown in many different 1-4244-0811-3/06/\$20.00 C2006 IEEE materials including GaAs [7-11], InAs [6,7], InP [10], InAs [11,12], GaP [10], Si [13] and AlGaAs [11,14]. Techniques have been developed that allow the precise control of the size, shape and position of nanowires grown on the substrate with an accuracy of nanometers [9]. Furthermore, the nanowires may be grown with uniform diameters over the length of the wire, which is important for quantum confinement properties. Nanowire heterostructures are formed by switching the growth materials [15]. Various heterostructures have been grown including $\mathrm{Si} / \mathrm{SiGe}$ [16], GaAs/GaP [17] and InP/InAs [15]. It has been demonstrated that devices with high quality dislocation-free material interfaces may be grown in InP/InAs systems [18]. This is achieved through a very slow growth process. The uniformity of nanowire diameter and defect free interfaces make the development of novel devices possible.

To fabricate devices, nanowires are removed from the growth substrate and placed on a $\mathrm{SiO}_{2}$ capped $\mathrm{Si}$ wafer [6]. Lithography is then used to fabricate contacts to wires.

Modulation doping has been demonstrated allowing nanowire p-n junctions [19] and light-emitting diodes [20, 21], photo-detectors [21], transistors [22], inverters [22] and biological nano-sensors [23] to be demonstrated. Resonant tunneling diodes [24], single-electron transistors [25] and few-electron quantum dots [26] have been demonstrated using nanowire heterostructures.

Measurements on nanowire composites [27, 28], nanowire arrays [29] and individual nanowires [30] have been performed investigating their thermoelectric properties. No measurements of the thermoelectric properties of nanowire heterostructures have been conducted.

\section{Background}

The figure of merit in bulk thermoelectrics has been relatively stagnant over the last forty years with $Z T \sim 1$ and efficiencies in commercial devices of about $10 \%$ of the Carnot limit [31]. Hicks and Dresselhaus proposed that the reduced dimensionality of nanowires could be utilized to enhance the electronic density of states in energy ranges advantageous to thermoelectric power generation [1]. They predicted $Z T \approx 6$ for $\mathrm{Bi}_{2} \mathrm{Te}_{3}$ nanowires compared to 2.5 and 0.5 for superlattice and bulk systems respectively.

Thermal-to-electric energy conversion with efficiency near the Carnot limit would require negligible lattice heat conduction and reversible electron transport between the hot and cold reservoirs, that is, current flow without entropy production. We recently showed [3] that the latter conditions can indeed be achieved in principle if the only electrons that can travel between the reservoirs are those that have a specific energy given by 


$$
E_{0}=\frac{\mu_{C} T_{H}-\mu_{H} T_{C}}{T_{H}-T_{C}}
$$

where $T_{H / C}$ and $\mu_{H / C}$ are the hot/cold reservoir temperatures and chemical potentials respectively. Electrons at this energy are said to be in 'energy-specific equilibrium' [3] because they can move reversibly between the two reservoirs. If electrons are transmitted at an energy infinitesimally close to $E_{0}$, the efficiency of energy conversion will approach the Carnot value.

We propose a resonant tunneling double barrier heterostructure nanowire device, as shown in Figs. 1 and 2, which filters electrons such that only those within a narrow range around $E_{0}$ are transmitted. The potential of the system may be varied in two ways, either by applying a source-drain bias or a gate voltage which varies the global potential of the device. Specific structural parameters will be proposed for an InP/InAs system. Due to a large lattice mismatch, bulk InP/InAs heterostructures may not be grown. It is possible to grow nanowire heterostructures using the two materials because of strain relaxation due to the small size of the crystal [6].

The InAs/InP conduction band offset has been estimated through thermionic emission experiments to be around 0.57 $\mathrm{eV}$ [18]. This value is used in calculations. The effective masses used are $0.08 m_{e}$ and $0.023 m_{e}$ for InP and InAs respectively [32]. Calculations are performed using a Landauer equation based method detailed elsewhere [33].

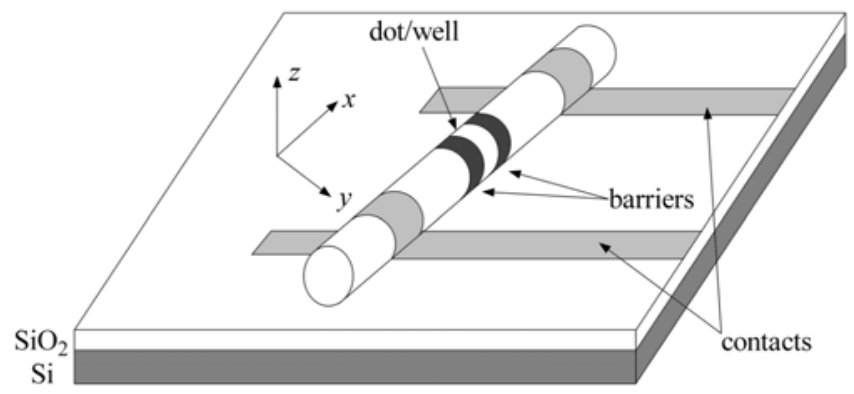

Figure 1: An illustration of quantum dot embedded resonant tunneling nanowire heterostructure on a $\mathrm{SiO}_{2}$ insulated $\mathrm{Si}$ wafer with contacts.

\section{Practical requirements for near-Carnot efficiency}

There are a number of requirements our system must meet in order to achieve an efficiency approaching the Carnot value.

First, the filtering of electron energies for transmission must occur according to their total energy. This is not possible in conventional 3D superlattices which filter electrons according to their energy in the direction of transport only, allowing energies in the transverse spatial dimensions to take on random values [34]. In a nanowire, electron energies are quantized in the transverse dimensions.

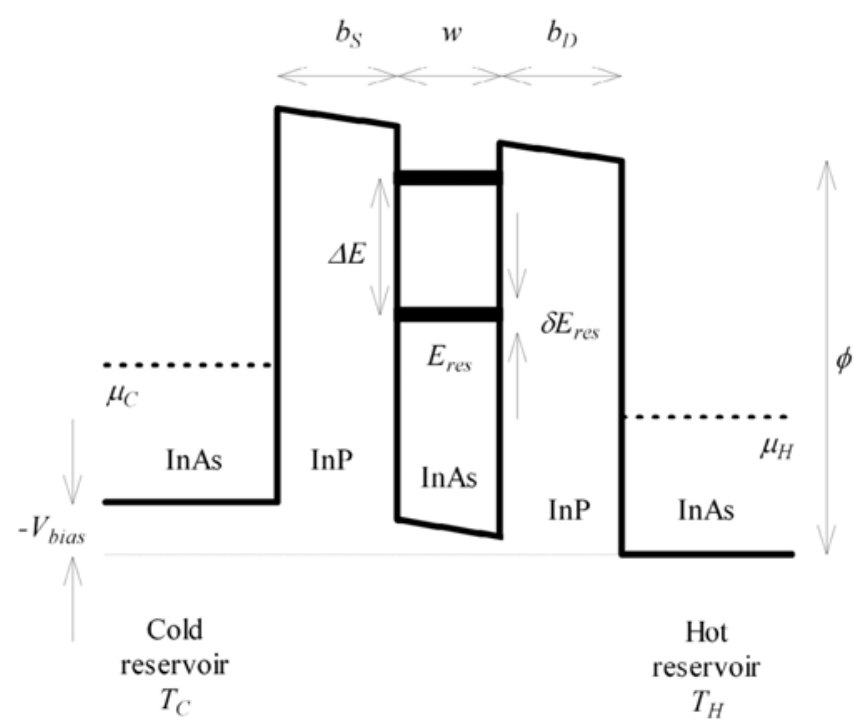

Figure 2: Band diagram showing the double barrier structure and key system parameters used in the model. $\mu_{H / C}$ are the chemical potentials of the hot/cold reservoirs, $T_{H / C}$ are the temperatures of the hot/cold reservoirs, $E_{\text {res }}$ is the resonant transmission energy and has energy width $\delta E_{\text {res }}, \Delta E$ is the energy gap between the first and second resonance (if any), $\varphi$ is the conduction band offset, $V_{\text {bias }}$ is the bias voltage on the system applied between the source and drain, and $b_{S}, b_{D}$ and $w$ are the source barrier, drain barrier and well widths, respectively.

Second, the double barrier needs to be designed such that the longitudinal electron energies are restricted to a narrow range. Efficiency is predicted to be higher for smaller $\delta E_{\text {res }}$ [35], which can be tuned by adjusting the barrier widths.

Third, one of the net resultant energy levels in the device must dominate electronic transport. Should the energy levels be sufficiently spaced relative to the thermal energy, $k_{B} T(\sim 1$ $\mathrm{meV}$ at $10 \mathrm{~K})$, transport in the nanowire attached to the double barrier will be restricted to a single level.

Fourth, heat leaked by the lattice must be very low if high efficiency is to be achieved. This is particularly important when $\delta E_{\text {res }}$ is small because then the electron current and the power of the device will be very low.

Fifth, the overall width of the double barrier structure should be less than the electron mean free path so that electrons are not scattered, but travel ballistically between the reservoirs.

As shall be demonstrated, a resonant tunneling quantum dot embedded nanowire system is capable of meeting these requirements.

\section{Energy levels in nanowires}

We will now analyze in detail the energy levels in a resonant tunneling nanowire heterostructure and discuss how the device must be structured in order to meet the stated requirements for near-Carnot efficiency. In a resonant tunneling nanowire device, there is an interplay between three effects that will determine the net energy level spacing. These are: (a) the transverse quantization due to the width of the 
nanowire, (b) the longitudinal quantization due to the levels in the potential well/quantum dot and (c) the charging energy caused by the capacitance of the quantum dot.

\section{Transverse energy levels}

The cross-sectional shape and diameter of the nanowire determine the transverse quantization energies in the $y$ and $z$ directions. InAs/InP nanowires have either a hexagonal or square cross section when grown in the $<111>$ and $<001>$ directions respectively [26]. Since the potential barrier due to the vacuum and $\mathrm{SiO}_{2}$ is high, it is accurate to consider the nanowire bounded by an infinite potential. The energy levels for hexagonal nanowires may be obtained through a numerical solution of the Schrödinger equation. Energy level solutions in the $y$ and $z$ directions for the square cross section are given by the standard infinite square well model; $E_{y / z}=\pi^{2} \hbar^{2} n_{y / z}^{2} / 2 m^{*} a_{y / z}^{2}$. The high vacuum $/ \mathrm{SiO}_{2}$ potential also means that we may accurately assume that the transverse energy levels are infinitely sharp. Fig. 3(a) shows the energy levels in one transverse dimension, for a square cross section nanowire of width $55 \mathrm{~nm}$. Two electrons may occupy each of these levels due to spin degeneracy. The total transverse energy is given by $E_{y}+E_{z}$, giving the energy level structure shown in Fig. 3(b). Four electrons are allowed to occupy some levels due to spin degeneracy combined with the symmetry of the square potential. The low effective mass of InAs gives large spacing between the energy levels. The transverse energy levels are closer for thinner nanowires. Although the details vary, the energy level structure is generally similar in form for nanowires with different crosssectional profiles.

\section{Longitudinal energy levels}

Longitudinal energies, in the direction of the wire, $x$, are quantized due to the double barrier resonant tunneling structure. Transmission through the double barrier is allowed at certain longitudinal energies corresponding to the bound states of the quantum dot. Electrons with energies above the barrier height will generally be transmitted unobstructed through the dot. The contribution of such electrons to the current should be negligible, however, due to the low occupation of states at such high energies, particularly at low temperatures.

The energy levels of the dot may be determined by numerically solving the Schrödinger equation for the finite width/finite potential barrier system. Fig. 4 shows the longitudinal energy levels calculated for devices with dot widths of $7 \mathrm{~nm}$ and $100 \mathrm{~nm}$. The narrower the dot is, the larger the spacing between longitudinal energy levels.

\section{Net quantum energy levels}

The net quantum energy levels in the dot are the results of the transverse confinement in the $y$ and $z$ directions by the width of the nanowire and the longitudinal confinement in the $x$ direction due to the width of the dot. Electrons will therefore be selected according to their total energy as required. The energy spread of electrons transmitted at any level may be restricted by increasing the width of the barriers

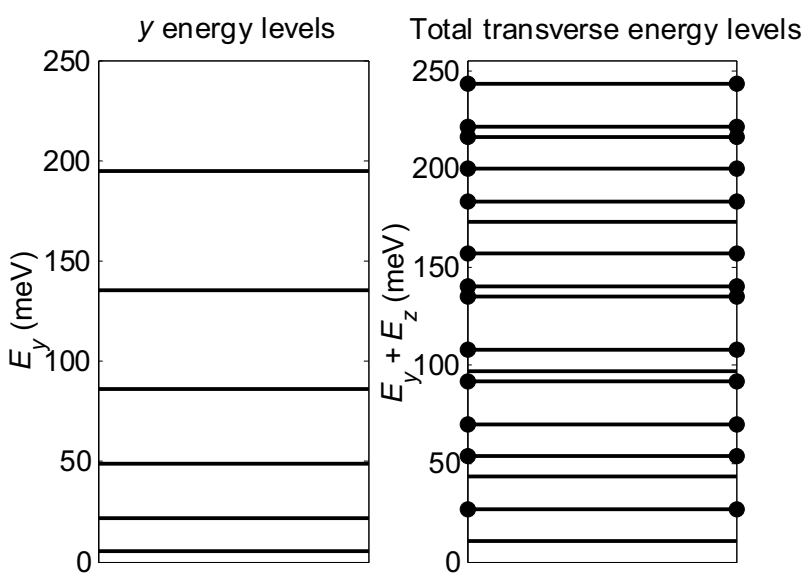

Figure 3: (a) The energy levels in one transverse dimension for a square cross section nanowire of width $55 \mathrm{~nm}$. (b) The resultant total transverse energy levels for the same nanowire when those in the $y$ and $z$ direction are combined. Circles indicate where two levels have the same energy.

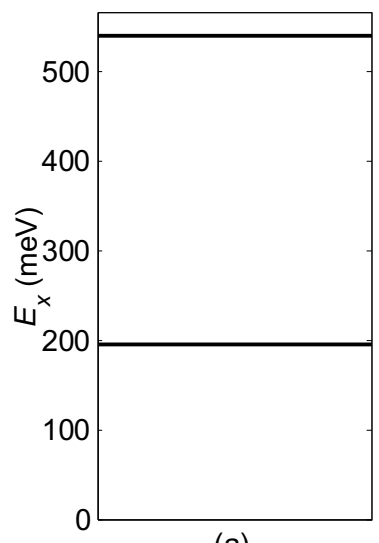

(a)

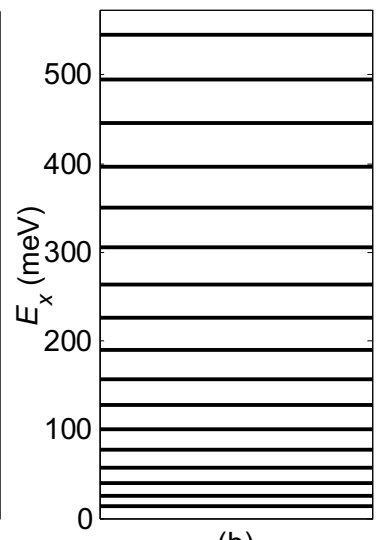

(b)
Figure 4: The longitudinal energy levels for a quantum dot of width (a) $7 \mathrm{~nm}$ and (b) $100 \mathrm{~nm}$ with barrier widths of $7 \mathrm{~nm}$.

defining the dot [33]. For each longitudinal energy (shown in Fig. 4) electrons may occupy any of the transverse energy levels (shown in Fig. 3). This gives the net quantum energy level structure shown in Fig. 5.

\section{Capacitance and charging energy}

Measurements on quantum-dot embedded nanowires show small capacitance and Coulomb blockade effects [6, 24-26]. This means that the spacing of the electron energy levels is increased by $\Delta E_{C}=e^{2} / C$ where $C$ is the system capacitance [36]. The origin of this additional energy gap is the electrostatic repulsion an electron in the nanowire reservoir feels due to the presence of an electron on the dot. Energy levels that previously allowed two or four electrons will therefore be split into distinct levels separated by $\Delta E_{C}$. Odd energy level gaps will always be equal to $\Delta E_{C}$. Every second or fourth energy gap will be given by $\Delta E_{C}+\Delta E_{N}$ where $\Delta E_{N}$ is the quantum spacing between the $N$ th and $(N-1)$ th energy levels. 


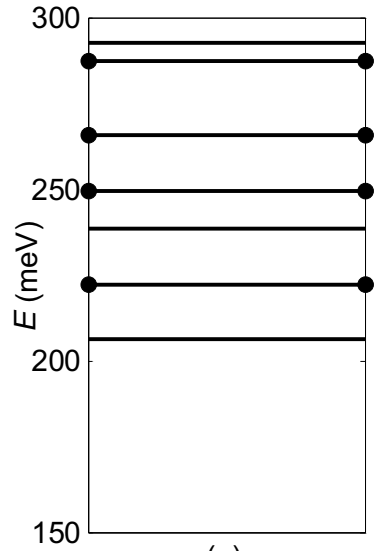

(a)

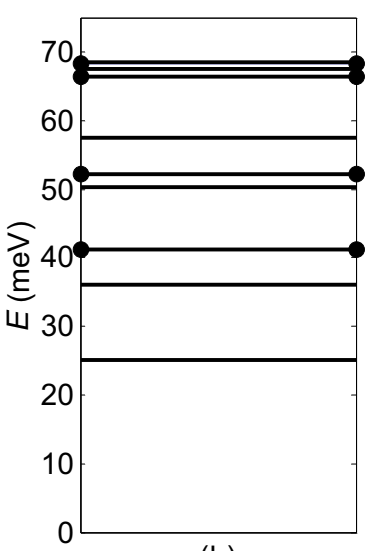

(b)
Figure 5: The net quantum energy levels for systems with nanowire width of $55 \mathrm{~nm}$, barrier widths of $10 \mathrm{~nm}$ and dot width of (a) $7 \mathrm{~nm}$ and (b) $100 \mathrm{~nm}$. Circles indicate two levels with the same energy.

Typically reported experimental values for $\Delta E_{C}$ range from $4 \mathrm{meV}$ to $6 \mathrm{meV}$ depending on system dimensions [26]. The total system capacitance, $C$, is given by the sum of the source, drain and gate capacitances. For any quantum dot embedded nanowire device, the charging energy and system capacitances may be determined in a relatively straight forward manner from its stability diagram. A stability diagram is a two dimensional plot of differential conductance, $d I / d V$, versus source drain voltage and gate voltage. The charging energies and capacitances are determined from the dimensions of the diamonds [26]. It has been shown that the plate capacitor formula, $C_{P}=\varepsilon_{r} \varepsilon_{0} A / t$, gives a reasonable estimate of the capacitances [25] and that the gate capacitance scales with dot width as this equation predicts [26]. To estimate the capacitance of nanowires with different configurations we will therefore scale the reported experimental values $[25,26]$ according to the plate capacitor formula

$$
C=1 a F \frac{w}{10 n m} \frac{d}{55 n m}+13 a F \frac{d^{2}}{(55 n m)^{2}} 5 \mathrm{~nm}\left(\frac{1}{b_{s}}+\frac{1}{b_{d}}\right)
$$

where $d$ is the diameter of the nanowire. Fig. 6 shows the final energy level spacing for the resonant tunneling nanowire device, with charging energies included. The spacing is much more regular than that of the net quantum levels. The charging energy for the smaller $7 \mathrm{~nm}$ dot is larger due to its lower capacitance. The smallest energy gap between transmission energies in such a Coulomb blockaded system will always be $\Delta E_{C}$.

\section{Device structure for an energy-selective thermoelectric}

It is now possible to discuss how the nanowire device should be structured in order to achieve an electron energy spectrum that meets the requirements described for near-Carnot efficiency. There are two ways the required energy

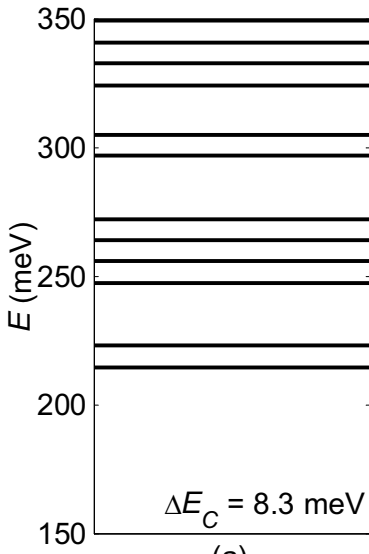

(a)

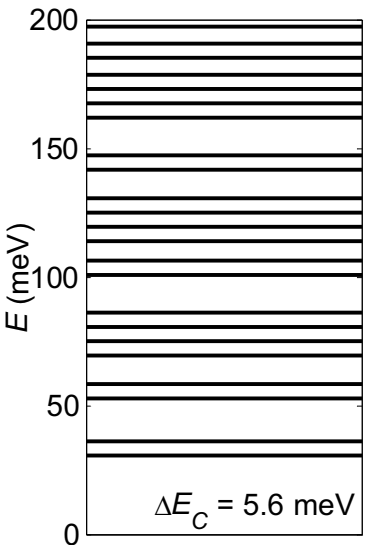

(b)
Figure 6: The net energy levels with charging effects for a device with dot width (a) $7 \mathrm{~nm}$ and (b) $100 \mathrm{~nm}$.

filtering could be achieved. The first is to have a very low charging energy, $\Delta E_{C}<k_{B} T$. In this limit, we would effectively have no splitting of the net quantum energy levels relative to the thermal energy. In this case, the quantum energy gap between the levels would need to be at least a few $k_{B} T$ to ensure a single transmission energy dominated transport. Realizing this limit requires very high capacitance, $C>6000 \mathrm{aF}$ at $10 \mathrm{~K}$. Based on Eq. 2, the capacitance can be increased by decreasing the barrier widths, increasing the dot width and increasing the wire diameter, none of which is desirable. Decreasing the barrier width increases the width of the electron energy spectrum reducing electronic efficiency. Increasing the dot width and wire diameter decrease the energy gap between the quantum energy levels that we require to be large in this instance.

The second way to achieve the required energy filtering is to utilize the charging energy to provide the energy level spacing. In this case, we require $\Delta E_{C} \gg k_{B} T$ and a small capacitance, the opposite to the previous case. Since $k_{B} T \sim 1$ $\mathrm{meV}$ at $10 \mathrm{~K}, \Delta E_{C}>5 \mathrm{meV}$ is desirable. This is equivalent to a system capacitance of less than $30 \mathrm{aF}$. This may be easily achieved with the InAs/InP nanowires discussed and the suitability of different configurations estimated using Eq. 2. In fact, this means that any nanowire with a strong Coulomb blockade effect might make a suitable candidate for the energy filtering of interest here.

Barriers of width $7 \mathrm{~nm}$ or larger should provide a sufficiently narrow electron energy spectrum. Since the system potential may be varied via the gate voltage, the magnitude of the energy level utilized is not critical and a dot of any width may be used so long as the charging energy is sufficient. It might be desirable in a particular experiment to use a narrow dot with strong quantum confinement, or conversely, a wide dot so that the energy level used is close to the Fermi level in the nanowire reservoirs. In any case, the gate voltage should be tuned such that suitable current is achieved through the desired energy level. 


\section{Proof-of-principle experiment}

Performing a proof-of-principle experiment to measure thermoelectric effects in energy filtering nanowires at low temperature $(T<10 \mathrm{~K})$ has a number of advantages. At low temperatures a lower charging energy is required and less efficiency reducing 'thermal smearing' of the electron energy spectrum occurs. Electron-phonon coupling is also very weak below $10 \mathrm{~K}$ [37]. In this case one can assume electronelectron interactions establish a quasi-equilibrium electron temperature that is independent of the phonon temperature. One electron reservoir can be heated using a heat current without phonon-mediated heat flow to the cold electron reservoir. Thus, high electronic efficiencies should be possible.

Determining the efficiency of the nanowire device requires measurements of the heat flow through the device, which could be difficult. It has been shown that with ideal electron energy filtering, the Seebeck coefficient approaches a theoretical maximum value given by $S_{0}=\left(E_{\text {res }}-\mu_{H}\right) / e T_{H}$. The Seebeck coefficient of the nanowire device may be determined more easily than efficiency, by simply measuring the temperature and electrical properties of the system. How close the measured Seebeck coefficient is to the $S_{0}$ will provide an indication as to whether or not the device is filtering the electron energy spectrum as desired. Fig. 7 shows that calculated electronic efficiency approaches the Carnot value and that as the width of the spectrum is decreased through widening the barriers, the Seebeck coefficient approaches $S_{0}$.

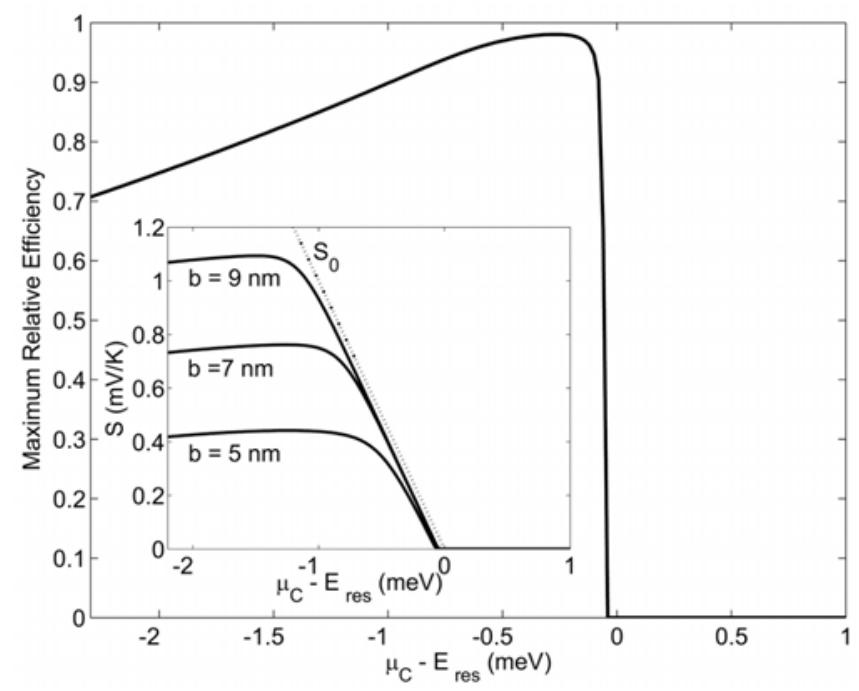

Figure 7: The electronic efficiency relative to the Carnot value of the nanowire thermoelectric device with $7 \mathrm{~nm}$ dot versus $\mu_{C}$ (with bias voltage tuned for maximum efficiency). Inset: the Seebeck coefficient, $S$, as a function of $\mu_{C}$ for different barrier widths as labeled. The maximum possible Seebeck coefficient, which is associated with reversibility, $S_{0}$, is also included.

Although phonon heat backflow would dominate, an experiment demonstrating idealized electron transport at room temperatures could also be performed. This would require very low capacitance, in the order of $1 \mathrm{aF}$, to provide the required separation between levels. Using a barrier width of $10 \mathrm{~nm}$ (barrier widths beyond this will result in very small currents) and evaluating Eq. 2 suggests that nanowires with diameters less than $15 \mathrm{~nm}$ would be required. In such a device, one would expect to observe Seebeck coefficients approaching $S_{0}$ at room temperature.

When performing an experiment, one should be aware that there will be a Seebeck coefficient associated with the contact between the three dimensional leads and the one dimensional wire. There will also be a resistance associated with this. Device currents will be very small, requiring precise calibration and measurement techniques.

\section{Conclusions}

We have discussed the requirements for a nanowire thermoelectric device to filter the electron energy spectrum such that high efficiencies approaching the Carnot value might be realized. An InP/InAs resonant tunneling heterostructure nanowire is capable of realizing these. A detailed analysis of the energy level structure in nanowires reveals that the device should be designed with capacitance lower than $\sim 30 \mathrm{aF}$ so that Coulomb blockade effects provide the required electron energy filtering. Finally, a proof-ofprinciple experiment was described, which it is hoped will measure a Seebeck coefficient revealing near-ideal electron energy filtering.

\section{Acknowledgments}

This work is supported by the Australian Research Council and the U.S. Office of Naval Research.

\section{References}

1. L. D. Hicks and M. S. Dresselhaus, "Thermoelectric figure of merit in a one-dimensional conductor," Phys. Rev. B, vol. 47, pp. R16631-R16634, 1993.

2. T. E. Humphrey, R. Newbury, R. P. Taylor, and H. Linke, "Reversible Quantum Brownian Heat Engines for Electrons," Phys. Rev. Lett., vol. 89, pp. 116801, 2002.

3. T. E. Humphery and H. Linke, "Reversible Thermoelectric Nanomaterials," Phys. Rev. Lett., vol. 94, pp. 096601, 2005.

4. L. Samuelson, M. T. Björk, K. Deppert, M. Larsson, B. J. Ohlsson, N. Panev, A. I. Persson, N. Skold, C. Thelander, and L. R. Wallenberg, "Semiconductor nanowires for novel one-dimensional devices," Physica E, vol. 21, pp. 560-567, 2004.

5. R. S. Wagner and W. C. Ellis, "Vapour-Liquid-Solid Mechanism of Single Crystal Growth," Appl. Phys. Lett., vol. 4, pp. 89-90, 1964.

6. C. Thelander, M. T. Björk, M. W. Larsson, A. E. Hansen, L. R. Wallenberg, and L. Samuelson, "Electron transport in InAs nanowires and heterostructure nanowire devices," Solid State Comm., vol. 131, pp. 573-579, 2004.

7. K. Hiruma, M. Yazawa, T. Katsuyama, K. Ogawa, K. Haraguchi, M. Koguchi, and H. Kakibayashi, "Growth and optical properties of nanometer-scale GaAs and InAs whiskers," J. Appl. Phys., vol. 77, pp. 447-462, 1995.

8. A. I. Persson, M. W. Larsson, S. Stenstrom, B. J. Ohlsson, L. Samuelson, and L. R. Wallenberg, "Solid-phase 
diffusion mechanisms for GaAs nanowire growth," Nat. Mat., vol. 3, pp. 677-681, 2004.

9. B. J. Ohlsson, M. T. Björk, M. H. Magnusson, K. Deppert, L. Samuelson, and L. R. Wallenberg, "Size-, shape- and position-controlled GaAs nano-whiskers," Appl. Phys. Lett., vol. 79, pp. 3335-3337, 2001.

10. T. Martensson, C. P. T. Svensson, B. A. Wacaser, M. W. Larsson, W. Seifert, K. Deppert, A. Gustafsson, L. R. Wallenberg, and L. Samuelson, "Epitaxial III-V Nanowires on Silicon," Nano Lett., vol. 4, pp. 1987-1990, 2004.

11. K. Haraguchi, K. Hiruma, M. Yazawa, and T. Katsuyama, "The Growth Mechanisms of Nanometer-scale GaAs, InAs and AlGaAs Whiskers," J. Electrochemical Soc., vol. 153, pp. C1-5, 2006.

12. B. J. Ohlsson, M. T. Bjork, A. I. Persson, C. Thelander, L. R. Wallenberg, M. H. Magnusson, K. Deppert, and L. Samuelson, "Growth and characterization of GaAs and InAs nano-whiskers and InAs/GaAs heterostructures," Physica E, vol. 13, pp. 1126-1130, 2002.

13. N. Ozaki, Y. Ohno, and S. Takeda, "Silicon nanowhiskers grown on a hydrogen-terminated silicon $\{111\}$ surface," Appl. Phys. Lett., vol. 73, pp. 3700-3702, 1998.

14.Z. H. Wu, X. Y. Mei, and H. E. Ruda, "Growth and photoluminescence characteristics of AlGaAs nanowires," Appl. Phys. Lett., vol. 85, pp. 657-659, 2004.

15. M. T. Björk, B. J. Ohlsson, T. Sass, A. I. Persson, C. Thelander, M. H. Magnusson, K. Deppert, L. R. Wallenberg, and L. Samuelson, "One-dimensional heterostructures in semiconductor nanowhiskers," Appl. Phys. Lett., vol. 80, pp. 1058-1060, 2002.

16. Y. Wu, R. Fan, and P. Yang, "Block-by-Block Growth of Single-Crystalline Si/SiGe Superlattice Nanowires," Nano Lett., vol. 2, pp. 83-86, 2002.

17. M. S. Gudisken, L. J. Lauhon, J. Wang, D. C. Smith, and C. M. Lieber, "Growth of nanowire superlattice structures for nanoscale photonics and electronics," Nature, vol. 404, pp. 974-977, 2002.

18. M. T. Björk, B. J. Ohlsson, T. Saas, A. I. Persson, C. Thelander, M. H. Magnusson, K. Deppert, L. R. Wallenberg, and L. Samuelson, "One-dimensional Steeplechase for Electrons Realized," Nano Lett., vol. 2, pp. 87-89, 2002.

19. K. Haraguchi, T. Karsuyama, K. Hiruma, and K. Ogawa, "GaAs p-n junction formed in quantum wire crystals," Appl. Phys. Lett., vol. 60, pp. 745-747, 1992.

20. K. Haraguchi, T. Katsuyama, and K. Hiruma, "Polarization dependence of light emitted from GaAs p-n junctions in quantum wire crystals," J. Appl. Phys., vol. 75, pp. 4220-4225, 1994.

21. J. Wang, M. S. Gudisken, X. Duan, Y. Cui, and C. M. Lieber, "Highly Polarized Photoluminescence and Photodetection from Single Indium Phosphide Nanowires," Science, vol. 293, pp. 1455-1457, 2001.

22. Y. Cui and C. M. Lieber, "Functional Nanoscale Electronic Devices Assembled Using Silicon Nanowire Building Blocks," Science, vol. 291, pp. 851-853, 2001.
23. Y. Cui, Q. Wei, H. Park, and C. M. Lieber, "Nanowire Nanosensors for Hightly Sensitive and Selective Detection of Biological and Chemical Species," Science, vol. 293, pp. 1289-1292, 2001.

24. M. T. Björk, B. J. Ohlsson, C. Thelander, A. I. Persson, K. Deppert, L. R. Wallenberg, and L. Samuelson, "Nanowire resonant tunneling diodes," Appl. Phys. Lett., vol. 81, pp. 4458-4460, 2002.

25. C. Thelander, M. T. Martensson, M. T. Björk, B. J. Ohlsson, M. W. Larsson, L. R. Wallenberg, and L. Samuelson, "Single-electron transistors in heterostructure nanowires," Appl. Phys. Lett., vol. 83, pp. 2052-2054, 2003.

26. M. T. Björk, C. Thelander, A. E. Hansen, L. E. Jensen, M. W. Larsson, L. R. Wallenberg, and L. Samuelson, "FewElectron Quantum Dots in Nanowires," Nano Lett., vol. 4, pp. 1621-1625, 2004.

27. J. P. Heremans, C. M. Thrush, D. T. Morelli, and M.-C. Wu, "Thermoelectric Power of Bismuth Nanocomposites," Phys. Rev. Lett., vol. 88, pp. 216801, 2002.

28. J. P. Heremans, C. M. Thrush, D. T. Morelli, and M.-C. $\mathrm{Wu}$, "Resistance, Magnetoresistance, and Thermopower of Zinc Nanowire Composites," Phys. Rev. Lett., vol. 91, pp. 076804, 2003.

29. Y.-M. Lin, O. Rabin, S. B. Cronin, J. Y. Ying, and M. S. Dresselhaus, "Semimetal-semiconductor transition in BiSb alloy nanowires and their thermoelectric properties," Appl. Phys. Lett., vol. 81, pp. 2403-2405, 2002.

30. J. Zhou, C. Jin, J. H. Soel, D. Li, and L. Shi, "Thermoelectric properties of individual electrodeposited bismuth telluride nanowires," Appl. Phys. Lett., vol. 87, pp. 113109-1-3, 2005.

31.H. J. Goldsmid, "Timeliness in the development of thermoelectric cooling," 17th Int. Conf. Thermoelectrics, pp. 25-28, 1998.

32. M. Levinshtein, S. Rumyantsec, and M. Shur, Handbook Series on Semiconductor Parameters, vol. 1. Singapore: World Scientific Publishing, 1996.

33. M. F. O'Dwyer, T. E. Humphery, and H. Linke, "Concept study for a high-efficiency nanowire based thermoelectric," Nanotechnology, vol. 17, pp. S338-S343, 2006.

34. D. Vashaee and A. Shakouri, "Electronic and thermoelectric transport in semiconductor and metallic superlattices," J. Appl. Phys., vol. 95, pp. 1233-1245, 2004.

35. M. F. O'Dwyer, R. A. Lewis, C. Zhang, and T. E. Humphrey, "Electronic efficiency in nanostructured thermionic and thermoelectric devices," Phys. Rev. B, vol. 72, pp. 205330-110, 2005.

36. H. Van Houten, C. W. J. Beenakker, and A. A. M. Staring, "Coulomb-Blockade Oscillations in Semiconductor Nanostructures," in Single Charge Tunneling, H. Grabert and M. H. Devoret, Eds. New York: Plenum Press, 1992.

37. M. L. Roukes, M. R. Freeman, R. S. Germain, R. C. Richardson, and M. B. Ketchen, "Hot Electrons and Energy Transport in Metals at Millikelvin Temperatures," Phys. Rev. Lett., vol. 55, pp. 422-425, 1985. 